\title{
Applying calcium fluoride and silica particles: A solution to improve color homogeneity of pc-WLEDS
}

\author{
Huu Phuc Dang1, Nguyen Thi Phuong Loan ${ }^{2}$, Thanh Tung Nguyen ${ }^{3}$, Sang Dang Ho \\ ${ }^{1}$ Faculty of Fundamental Science, Industrial University of Ho Chi Minh City, Vietnam \\ ${ }^{2}$ Faculty of Fundamental 2, Posts and Telecommunications Institute of Technology, Vietnam \\ ${ }^{3}$ Institute Applied of Technology, Thu Dau Mot University, Thu Dau Mot City, Binh Duong province, Vietnam \\ ${ }^{4}$ Faculty of Electrical and Electronics Engineering, Ton Duc Thang University, Ho Chi Minh City, Vietnam
}

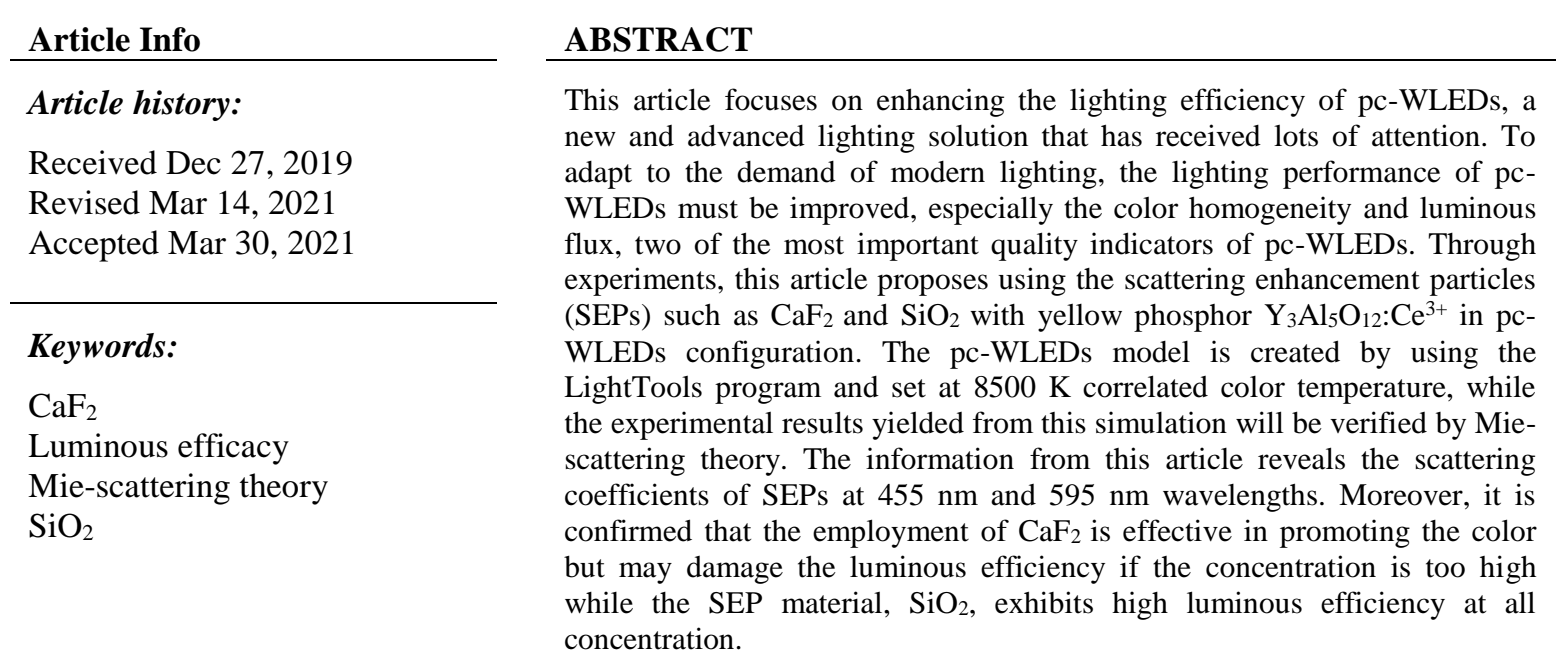

This is an open access article under the CC BY-SA license.

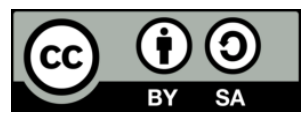

\section{Corresponding Author:}

Sang Dang Ho

Faculty of Electrical and Electronics Engineering

Ton Duc Thang University

No. 19 Nguyen Huu Tho Street, Tan Phong Ward, District 7, Ho Chi Minh City, Vietnam

Email: hodangsang@tdtu.edu.vn

\section{INTRODUCTION}

The light-emitting diodes (LEDs) have been being applied in both indoor and outdoor occasions recently [1]. The LEDs with outstanding characteristics, such as energy efficiency, cost effectiveness, high endurance, and low waste emission rate, have earned it the reputation in the lighting industry and is expected to replace older lighting solutions such as incandescent bulbs and discharge lamps [2]. The common white LED, also known as phosphor-converted white LED (pc-WLED), is created by integrating colors, a process that combines the blue light (InGaN) from the blue chips and the emitted yellow light that is converted from the blue light by phosphor [3], [4]. Although the use of pc-WLEDs can still be spread to more advanced field, the quality of pc-LEDs must be improved, particularly the light extraction proficiency [5]. Many studies were conducted and proposed different ideas, and one of them is adjusting the packaging by comparing conformal, using hemisphere glass, or employing the remote phosphor structure [6]. Others are the correlated color temperature (CCT), which is a decisive factor to the light output of WLED, and the impact of phosphor particles size and particle number [7], [8]. To study the aspects mentioned above, a process named Monte 
Carlo Ray was utilized to simulate the pc-LED model for the research of scattering effect, absorption coefficient, conversion efficiency, packaging arrangement, and the index of refraction of the phosphor material and polymer of the WLED design [9].

This simulated model was successfully applied with different types of phosphor (YAG, Silicate, and green YAG) and able to measure all the parameters that affect the lighting quality of pc-LEDs, including the light loss from backscattering happens during the phosphor emission process [10], [11]. The color uniformity is one of the important indicators that can influence the quality of light yielded from the pc-LEDs; however, it did not receive enough attention as it should in research [12]. The lack of color uniformity is usually cause by central and directional blue source combined with phosphor particles having an isotropic scattering that results in a phenomenon called the yellow ring, which impairs the color quality of pc-LEDs and causes irritation for the eyes [13]. This is due to the blue lights at high CCT assemble in the ordinary direction while the yellow lights gather in the peripheral direction, thus, creating the imbalance of light at these directions. The angular CCT deviation (DCCTD) for non-optimized configuration can go up to $3000 \mathrm{~K}$ and even higher in larger size applications used for lighting on a surface [14]. Many solutions to control the DCCTD were proposed in recent researches from simple ideas such as adjusting the method of conformal coating, applying specialized lens, and re-design the phosphor layer to a more advanced method of applying a patterned sapphire substrate or a multi-layer phosphor package in which the phosphor films are arranged based on their refractive indices [15]-[19]. The influences of these methods are not the same and should be regarded alongside the changes in luminous flux and the CCT [20]. Comparing a low CCT pc-LEDs with higher phosphor scattering to a high CCT pc-LEDs with lower scattered phosphor, the reduction of DCCTD is harder to achieve and more desirable. Chen et al. [21] combining $\mathrm{ZrO}_{2}$ Nano-particles to the phosphor layer in order to accomplish the DCCTD decrease. Using nano-particles $\mathrm{ZrO}_{2}$ in remote phosphor structure was proved to also boost the backscattering effect of $\mathrm{ZrO}_{2}$, however, the phosphor reduction was not mentioned in this finding. The increase in backscattering effect means the recycling of photon is also higher, therefore, sometimes can be good for the improvement of lumen output. However, this result is achieved only by comparing the luminous flux and then concluding on the impact of zirconia $\left(\mathrm{ZrO}_{2}\right)$ without regarding to the fluctuation of CCT during the experiment, therefore, it is difficult to determine the accuracy of the result.

In this article, we aim to achieve the reduction of DCCTD in high CCT devices by incorporating calcium fluoride $\left(\mathrm{CaF}_{2}\right)$ and silica $\left(\mathrm{SiO}_{2}\right)$ particles into the phosphor packaging. The model used in the experiments of this research is the most commonly used configuration, the pc-WLED with hemisphere package. The results from the experiments and the model are both studied and presented regarding to the CCT. Besides the color uniformity, the influence of using $\mathrm{CaF}_{2}$ and $\mathrm{SiO}_{2}$ in the phosphor layer at each CCT in accordance with different concentrations and particle sizes is also one of the analyzed points. The research results show that controlling the concentration and particle size of $\mathrm{CaF}_{2}$ and $\mathrm{SiO}_{2}$ can enhance both color quality and lumen output.

\section{ANALYZATION ON THE SCATTERING EFFECT}

The scattering enhancement particles (SEPs) are the source of light scattering effect. According to Mie-scattering theory, the presence of SEPs in conformal phosphor pc-WLED structure enables the scattering effect, which will be measured by MATLAB [21]-[25]. The important values of scattering coefficient $\mu_{\text {sca }}(\lambda)$, the anisotropy factor $g(\lambda)$, the reduced scattering coefficient $\delta_{\text {sca }}(\lambda)$ and the scattering amplitude functions $S_{l}(\theta)$ and $S_{2}(\theta)$ are calculated using the (1) presented:

$$
\mu_{s c a}(\lambda)=\int N(r) C_{s c a}(\lambda, r) \mathrm{d} r
$$

In (1), $N(r)$ represents the diffusional particles in each cubic millimeter that can also be defined as diffusional density distribution, while $C_{s c a}$ indicates the scattering cross section $\left(\mathrm{mm}^{2}\right)$. The wavelength $(\mathrm{nm})$ is shown as $\lambda$, and SEPs particles radius (mm) is $r$.

In Figure 1 is the graph presenting the scattering coefficients of $\mathrm{CaF}_{2}$ and $\mathrm{SiO}_{2}$. The results of Figure 1 suggest that the changes in scattering coefficients depend on the wavelength, which is expressed in the wavelength band from $380 \mathrm{~nm}$ to $780 \mathrm{~nm}$ in both phosphors. Overall, the scattering coefficient put out by $\mathrm{CaF}_{2}$ phosphor are higher than that of $\mathrm{SiO}_{2}$, which explains for the better color quality achieved in phosphor structure with phosphor $\mathrm{CaF}_{2}$. Having high scattering coefficient means there are more scattering processes happened within the pc-WLEDs model before the light is ejected. The more scattering processes occur, the better light rays are mixed, which lower the color deviation and lead to greater color quality. The luminous flux, on the other hand, is a completely different parameter to the color homogeneity one, and it might not benefit from high scattering coefficient. Developing pc-WLEDs based on these traits of color quality and luminous flux is essential as these traits are applied for all devices with phosphor particles. The most 
common method to adjust the color quality and luminous flux is changing the concentration of phosphor, in this case are the concentrations of $\mathrm{CaF}_{2}$ and $\mathrm{SiO}_{2}$, along with studying the impacts of phosphor particles size.

The content of Figure 2 analyzes the pc-WLEDs simulated by LightTools 8.7 program. The simulation of the pc-WLED is identical to the real WLEDs regarding all the components and parameters; therefore, it is suitable to use in optical properties study. The structure of the pc-WLED can be describes as follows: blue chips are placed at the bottom, the reflectors are on the sides, the mixture of yellow phosphor and the SEPs covers the blue chip, and a silicone lens on top of the pc-WLED. The size of the simulation is $2.1 \mathrm{~mm}$ in depth, $8 \mathrm{~mm}$ inner with a $10 \mathrm{~mm}$ reflector. The components of the simulation are 9 blue chips, one $0.08 \mathrm{~mm}$ phosphor layer, and $0.5 \mu \mathrm{m}$ spherical SEPs particles with 1.54 and 1.44 refractive indexes for $\mathrm{SiO} 2$ and $\mathrm{CaF} 2$, respectively. In addition, the yellow phosphor grain has $7.25 \mu \mathrm{m}$ average radius and 1.83 refractive index at all wavelength in the visible spectrum. The silicone glue used to mix the phosphors has fixed refractive index of 1.5. To maintain the CCT or ensure the lighting efficacy, the particles density distribution can be changed.

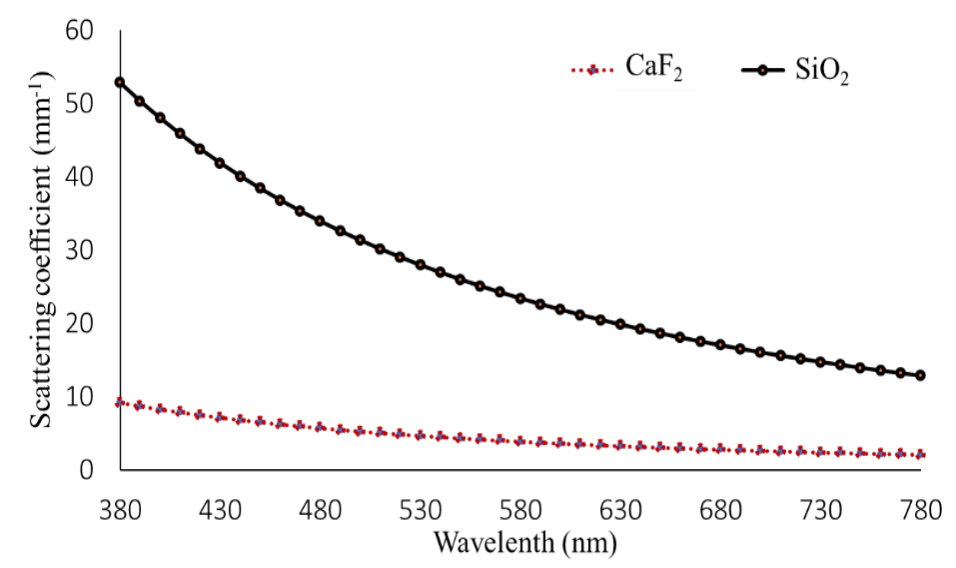

Figure 1. The scattering coefficient of SEPs at respective wavelength

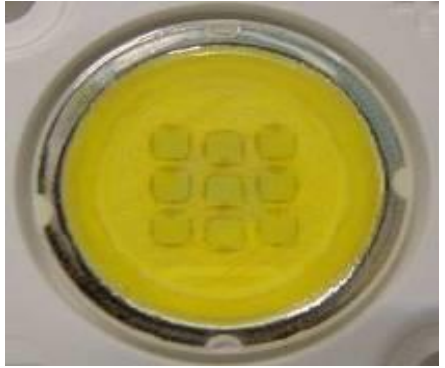

(a)

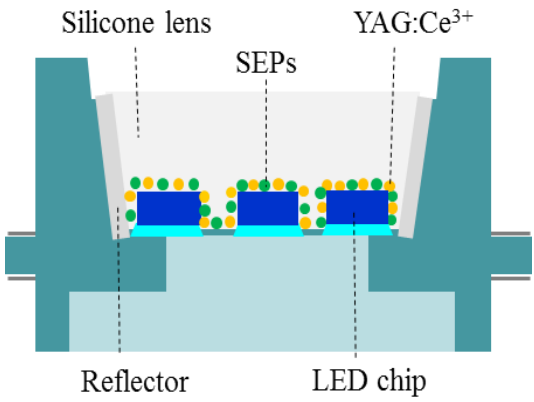

(c)
Lead frame: $4.7 \mathrm{~mm}$ Jentech Size-S

LED chip: $\mathrm{V} 45 \mathrm{H}$

Die attach: Sumitomo 1295SA

Gold Wire: 1.0 mil

Phosphor: ITC NYAG4_EL

(b)

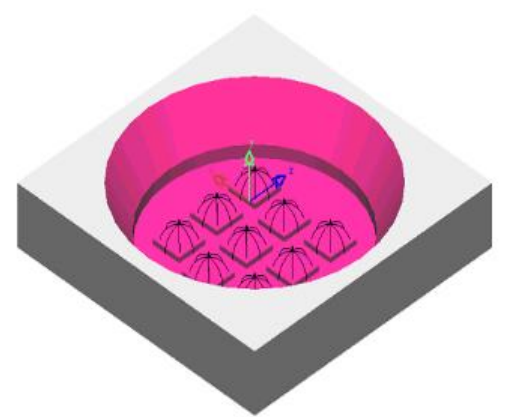

(d)

Figure 2. These figures are; (a) physical model of WLED sample, (b) WLEDs parameters, (c) illustration of WLEDs model cross section, (d) the simulated WLEDs model 


\section{RESULTS AND ANALYSIS}

To execute the experiments in this research the CCT must be stable by balancing the weight percentage of SEPs, the silicone, and the yellow phosphor. That means the concentration of yellow phosphor and SEPs must change regarding each other, which can be monitored and calculated with the (2).

$$
W_{\text {phosphor }}+W_{\text {silicone }}+W_{S E P}=100 \%
$$

In (2), $W_{\text {phosphor }}, W_{\text {silicone, }}$ and $W_{S E P}$ each represents the weight percentage of the yellow phosphor, the silicon, and SEPs. This percentage composition equation of pc-WLEDs provides management over the weight percentage components for keeping the CCT at $8500 \mathrm{~K}$. The CCT deviation is an important index because it affects the lighting performance of pc-WLEDs. If the CCT deviation is high, the light color at different angles will be different, and thus, leading to the yellow ring phenomenon and impairing the lighting performance of pc-WLEDs. Therefore, including CCT deviation in the study is important, and the CCT deviation can be computed with the (3).

$$
\Delta \mathrm{CCT}=\mathrm{CCT}_{(\mathrm{Max})}-\mathrm{CCT}_{(\mathrm{Min})}
$$

In which, $\mathrm{CCT}_{(\mathrm{Max})}$ is the highest $\mathrm{CCT}$ at $0^{\circ}$ viewing angle while $\mathrm{CCT}_{(\mathrm{Min})}$ demonstrates the lowest CCT at the viewing angle of $90^{\circ}$. The changes in these optical properties in pc-WLEDs are caused by the dissimilar particles emission spectra within the configuration. Trying to match the emitted blue light spectrum with the spectra of other light sources to minimize the CCT deviation is an effective way to address the issue. Observing the CCT deviation recorded from phosphor structure with $\mathrm{CaF}_{2}$ and $\mathrm{SiO}_{2}$ in Figure 3, we can see that $\mathrm{CaF}_{2}$ achieves the lowest index in deviation of angular amplitude than the other suggested SEP does at wavelength range of $400 \mathrm{~nm}$ and $800 \mathrm{~nm}$, which suggests that $\mathrm{CaF}_{2}$ is the more effective SEP to control the color deviation. The increase in emitted blue light to a sufficient amount is good for the color homogeneity of pc-WLEDs as the redundant yellow lights that causes the yellow ring will be absorbed. However, the blue light must not be redundant or insufficient because it will worsen the CCT deviation. $\mathrm{CaF}_{2}$ angular scattering amplitude is also high at $455 \mathrm{~nm}$ and $595 \mathrm{~nm}$ wavelength, which is similar to other SEP. In Figure 3, the $\mathrm{CaF}_{2}$ and $\mathrm{SiO}_{2}$ have almost triple the amount of $455 \mathrm{~nm}$ and $595 \mathrm{~nm}$ angular scattering amplitudes, compared to other wavelengths. This means adding SEPs to pc-WLEDs benefits color homogeneity and luminous, which can be verified by the $1800 \mathrm{~K} \mathrm{CCT}$ deviation reduction when comparing pc-WLEDs without SEPs at $2670 \mathrm{~K}$ to pc-WLEDs with SEPs. On the contrary, the concentration of $\mathrm{CaF}_{2}$ and $\mathrm{SiO}_{2}$ particles on their own will cause an increase in CCT deviation.

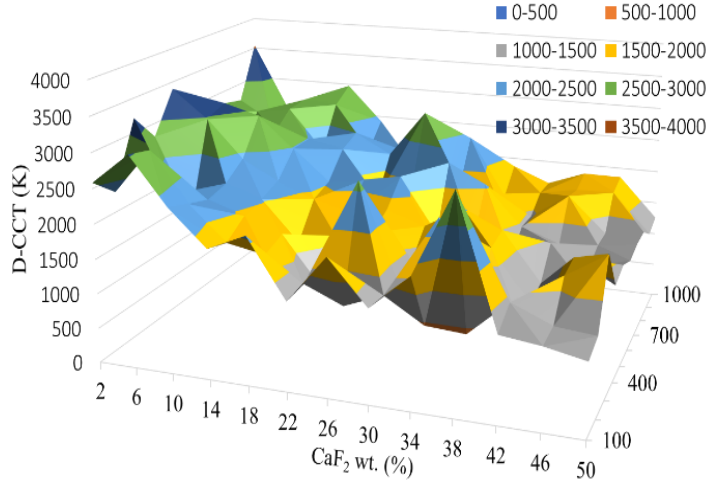

(a)

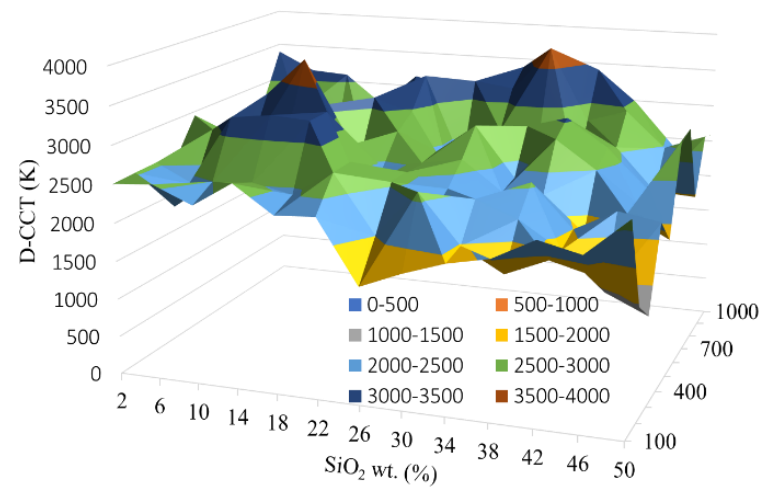

(b)

Figure 3. Comparison of the CCT deviations between the pc-LEDs using (a) $\mathrm{CaF}_{2}$ and (b) $\mathrm{SiO}_{2}$

Figure 4 presents the luminous flux of pc-WLEDs with the addition of $\mathrm{CaF}_{2}$ and $\mathrm{SiO}_{2} \mathrm{SEPs}_{\text {particles. }}$ The results of Figure 4 cover the ranges of SEPs concentrations and particle sizes from $0 \%$ to $50 \%$ and from $100 \mathrm{~nm}$ to $1000 \mathrm{~nm}$, respectively. Regarding the luminous flux results of $\mathrm{CaF}_{2}$ expressed in Figure 4(a), the luminous flux increases with concentration of $\mathrm{CaF}_{2}$ in the range from $0 \%$ to $20 \%$. However, it begins to decline once the concentration of $\mathrm{CaF}_{2}$ passes $20 \%$ and grows higher. The $\mathrm{SiO}_{2}$ luminous flux presented in Figure 4(b) shows that this SEP is favorable for the development of the light output as the achieved luminous 
flux increases with its concentration and particles size. These results confirm the importance of the particles size in reducing the light loss caused by backscattering effect, therefore, managing this element is also advisable. To study the amount of light energy loss from the excessive SEP concentration in $\mathrm{CaF}_{2}$, we can use the Lambert-Beer law and the Mie-scattering theory following the equation presented.

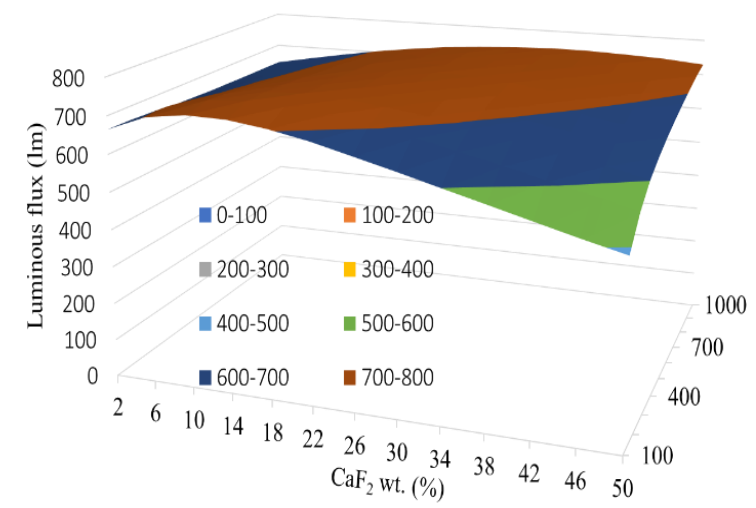

(a)

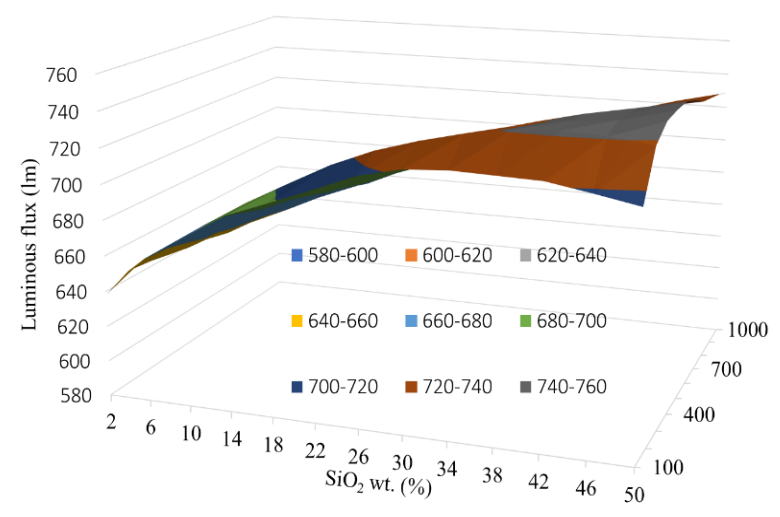

(b)

Figure 4. Comparison of luminous flux of pcLEDs adding (a) $\mathrm{CaF}_{2}$ and (b) $\mathrm{SiO}_{2}$

The Mie-theory is employed to estimate the scattered phosphor particles. Therefore, the scattering cross section $C_{s c a}$ in spherical particles can be calculated using the following equation with the parameter of transmitted light power calculated with the Lamber-Beer law:

$$
I=I_{0} \exp \left(-\mu_{e x t} L\right)
$$

The incident light power is $I_{0}$, and $L$ presents the phosphor film's thickness (mm). $\mu_{e x t}$ is known as the extinction coefficient, and calculated from the equation: $\mu_{e x t}=N_{r} . C_{e x t}$, where $N_{r}$ indicates the particles' number density distribution $\left(\mathrm{mm}^{-3}\right) . \mathrm{C}_{\mathrm{ext}}\left(\mathrm{mm}^{2}\right)$ demonstrates the extinction cross-section of phosphor particles. From the results of (4), which regards the lighting efficiency of pc-WLEDs, it can be assumed that with the SEPs concentration gradually increases, the luminous flux slowly falls off. This is due to the increased light scattering processes and the high SEPs concentration amplifies the light loss from backscattering effect, therefore, the emission energy is damaged.

\section{CONCLUSION}

With the aim to improve the lighting quality of pc-WLEDs configuration, the research of this article focus on the changes that SEPs, specifically $\mathrm{CaF}_{2}$ and $\mathrm{SiO}_{2}$, bring to color uniformity and luminous flux. After going through experiments and being verified by Mie-scattering theory together with the Monte Carlo simulation, the results confirm that the addition of SEPs is good for pc-WLED enhancement, and each type of SEPs has a different benefit. Because the effect of each SEPs is distinct and the efficiency relies on the concentration of phosphor, an optimal setting, which is in connection with the type of SEPs and the exact concentration for each application, is essential for further developments of pc-WLEDs. In this research, we have found that adding $\mathrm{CaF}_{2}$ and $\mathrm{SiO}_{2}$ can provide the control over the CCT deviation, an index that impacts the fluctuation of CCT. By managing the concentration of $\mathrm{CaF}_{2}$ phosphor, the CCT deviation can decrease to the lowest value while avoid damaging the luminous flux due to the excessive phosphor concentration. $\mathrm{SiO}_{2}$ is the phosphor material that can guarantee the growth of luminous flux regardless of the concentration. These findings are valuable for the manufacturing of pc-WLEDs with unique optical properties and can be served as a practical direction for further studies.

\section{ACKNOWLEDGEMENTS}

This research is funded by the Industrial University of Ho Chi Minh City under grant number: 21/1.3CB01. 


\section{REFERENCES}

[1] J. Cheng et al., "Photoluminescence properties of $\mathrm{Ca}_{4} \mathrm{La}_{6}\left(\mathrm{SiO}_{4}\right)_{4}\left(\mathrm{PO}_{4}\right)_{2} \mathrm{O}_{2}$-based phosphors for wLEDs," Chin. Opt. Lett., vol. 17, 2019, Art. no. 051602.

[2] O. H. Kwon et al., "White luminescence characteristics of red/green silicate phosphor-glass thick film layers printed on glass substrate," Opt. Mater. Express, vol. 6, no. 3, pp. 938-945, 2016, doi: 10.1364/OME.6.000938.

[3] Y. S. Qi et al., "Energy transfer between $\mathrm{Ce}^{3+}$ and $\mathrm{Sm}^{3+}$ in $\mathrm{Zn}_{2} \mathrm{GeO}_{4}$ phosphor with the native defects for lightemitting diodes," Chin. Opt. Lett., vol. 15, no. 8, 2017, Art. no. 081601, doi: 10.3788/COL201715.081601.

[4] Z. Huang et al., "Excellent optical thermometry based on upconversion emission in $\mathrm{SrMoO}_{4}: \mathrm{Er}^{3+}$ phosphor," Opt. Mater. Express, vol. 7, no. 7, pp. 2404-2410, 2017, doi: 10.1364/OME.7.002404.

[5] X. Liu et al., "Upconversion luminescence, intrinsic optical bistability, and optical thermometry in $\mathrm{Ho}^{3+/} \mathrm{Yb}^{3+}: \mathrm{BaMoO}_{4}$ phosphors," Chin. Opt. Lett., vol. 17, no. 11, 2019, Art. no. 111601.

[6] Y. Du et al., "Electroluminescent Properties of WLEDs With the Structures of Ce:YAG Single Crystal/Blue Chip and $\mathrm{Sr}_{2} \mathrm{Si}_{5} \mathrm{~N}_{8}: \mathrm{Eu}^{2+} / \mathrm{Ce}: \mathrm{YAG}$ Single Crystal/Blue Chip," Journal of Display Technology, vol. 12, no. 4, pp. 323-327, 2016.

[7] J. K. Sheu et al., "Warm-white light-emitting diode with high color rendering index fabricated by combining trichromatic InGaN emitter with single red phosphor," Optics Express, vol. 23, no. 7, pp. A232-A239, 2015, doi: 10.1364/OE.23.00A232.

[8] J. W. Moon et al., "Optical characteristics and longevity of the line-emitting $\mathrm{K}_{2} \mathrm{SiF}_{6}: \mathrm{Mn}^{4+}$ phosphor for LED application," Opt. Mater. Express, vol. 6, no. 3, pp. 782-792, 2016, doi: 10.1364/OME.6.000782.

[9] A. Liu et al., "Red-blue-green solid state light sources using a narrow line-width green phosphor," Optics Express, vol. 23, no. 7, pp. A309-A315, 2015, doi: 10.1364/OE.23.00A309.

[10] V. K. Rai and A. Pandey, "Efficient Color Tunable $\mathrm{ZnWO}_{4}: \mathrm{Er}^{3+}-\mathrm{Yb}^{3+}$ Phosphor for High Temperature Sensing," Journal of Display Technology, vol. 12, no. 11, pp. 1472-1477, 2016, doi: 10.1109/JDT.2016.2602503.

[11] C. Hu et al., "YAG:Ce/(Gd,Y)AG:Ce dual-layered composite structure ceramic phosphors designed for bright white light-emitting diodes with various CCT," Optics Express, vol. 23, no. 14, pp. 18243-18255, 2015, doi: 10.1364/OE.23.018243.

[12] Z. Z. Zhau et al., "White light obtainment via tricolor luminescent centers and energy transfer in $\mathrm{Ca}_{3} \mathrm{ZrSi}_{2} \mathrm{O} 9: \mathrm{Eu}^{3+}, \mathrm{Bi}^{3+}, \mathrm{Tb}^{3+}$ phosphors," Opt. Mater. Express, vol. 8, no. 11, pp. 3526-3542, 2018, doi: 10.1364/OME.8.003526.

[13] X. P. Li et al., "Highly stable and tunable white luminescence from Ag-Eu3+ co-doped fluoroborate glass phosphors combined with violet LED," Optics Express, vol. 26, no. 2, pp. 1870-1881, 2018, doi: 10.1364/OE.26.001870.

[14] C. M. Lee et al., "Gigabit-per-second white light-based visible light communication using near-ultraviolet laser diode and red-, green-, and blue-emitting phosphors," Optics Express, vol. 25, no. 15, pp. 17480-17487, 2017, doi: 10.1364/OE.25.017480.

[15] B. Li et al., "High-efficiency cubic-phased blue-emitting $\mathrm{Ba}_{3} \mathrm{Lu}_{2} \mathrm{~B}_{6} \mathrm{O}_{15}: \mathrm{Ce}^{3+}$ phosphors for ultraviolet-excited white-light-emitting diodes," Opt. Lett., vol. 43, no. 20, pp. 5138-5141, 2018, doi: 10.1364/OL.43.005138.

[16] J. Hu et al., "Near ultraviolet excited $\mathrm{Eu}^{3+}$ doped $\mathrm{Li}_{3} \mathrm{Ba}_{2} \mathrm{La}_{3}\left(\mathrm{WO}_{4}\right)_{8}$ red phosphors for white light emitting diodes," Opt. Mater. Express, vol. 6, no. 1, pp.181-190, 2016, doi: 10.1364/OME.6.000181.

[17] L. P. Li et al., "Roller coaster"-like thermal evolution of the $\mathrm{Er}^{3+}$ ion's red photoluminescence in $\mathrm{CaWO}_{4}: \mathrm{Yb}^{3+} \mathrm{Er}^{3+}$ phosphors," Opt. Lett., vol. 44, no. 17, pp. 4411-4414, 2019, doi: 10.1364/OL.44.004411.

[18] Y. Peng et al., "Luminous efficacy enhancement of ultraviolet-excited white light-emitting diodes through multilayered phosphor-in-glass," Appl. Opt., vol. 55, no. 18, pp. 4933-4938, 2016, doi: 10.1364/AO.55.004933.

[19] Z. C. Wen et al., "Fabrication and optical properties of $\mathrm{Pr}^{3+}$-doped $\mathrm{Ba}(\mathrm{Sn}, \mathrm{Zr}, \mathrm{Mg}, \mathrm{Ta}) \mathrm{O}_{3}$ transparent ceramic phosphor," Opt. Lett., vol. 43, no. 11, pp. 2438-2441, 2018, doi: 10.1364/OL.43.002438.

[20] P. Q. Jiang et al., "Thermally stable multi-color phosphor-in-glass bonded on flip-chip UV-LEDs for chromaticitytunable WLEDs," Appl. Opt. ,vol. 56, no. 28, pp. 7921-7926, 2017, doi: 10.1364/AO.56.007921.

[21] S. Sun et al., "Energy transfer between $\mathrm{Ce}^{3+}$ and $\mathrm{Tb}^{3+}$ and the enhanced luminescence of a green phosphor $\mathrm{SrB}_{2} \mathrm{O}_{4}: \mathrm{Ce}^{3+}, \mathrm{Tb}^{3+}, \mathrm{Na}^{+}$," Opt. Mater. Express, vol. 6, no. 4, pp. 1172-1185, 2016, doi: 10.1364/OME.6.001172.

[22] C. G. Zhang et al., "Mn ${ }^{4+}$-doped fluoride phosphors rapidly synthesized by ball milling," Opt. Mater. Express, vol. 8, no. 1, pp. 73-81, 2018, doi: 10.1364/OME.8.000073.

[23] Z. J. Zhang and W. C. Yang, "Tunable photoluminescence in $\mathrm{Ba}_{1-\mathrm{x}} \mathrm{Sr}_{\mathrm{x}} \mathrm{Si}_{3} \mathrm{O}_{4} \mathrm{~N}_{2}: \mathrm{Eu}^{2+} / \mathrm{Ce}^{3+}, \mathrm{Li}^{+}$solid solution phosphors induced by linear structural evolution," Opt. Mater. Express, vol. 9, no. 4, pp.1922-1932, 2019, doi: 10.1364/OME.9.001922.

[24] Q. Shao et al., "Broadband near-infrared light source derived from $\mathrm{Cr}^{3+}$-doped phosphors and a blue LED chip," Opt. Lett., vol. 43, no. 21, pp. 5251-5254, 2018, doi: 10.1364/OL.43.005251.

[25] Z. Hu et al., "Influence of co-doping $\mathrm{Si}$ ions on persistent luminescence of $\mathrm{ZnGa}_{2} \mathrm{O}_{4}: \mathrm{Cr}^{3+}$ red phosphors," Opt. Mater. Express, vol. 6, no. 4, pp. 2789-2818, 2016, doi: 10.1364/OME.6.001329. 